\title{
Liver transplantation for unresectable colorectal metastasis: a new hope
}

\author{
Andre Gorgen, Tommy Ivanics, Gonzalo Sapisochin \\ Multi-Organ Transplant Program, Division of General Surgery, University Health Network, Toronto General Hospital, Toronto, Canada \\ Correspondence to: Andre Gorgen. Multi-Organ Transplant Program, Division of General Surgery, University Health Network, Toronto General \\ Hospital, Toronto, Canada. Email: andre.gorgen@uhn.ca. \\ Comment on: Dueland S, Syversveen T, Solheim JM, et al. Survival Following Liver Transplantation for Patients With Nonresectable Liver-only \\ Colorectal Metastases. Ann Surg 2020;271:212-8.
}

Submitted Dec 20, 2019. Accepted for publication Jan 08, 2020.

doi: 10.21037/hbsn.2020.01.04

View this article at: http://dx.doi.org/10.21037/hbsn.2020.01.04

Despite recent advances in medical and surgical treatments, surgical resection remains the only curative option for patients with colorectal liver metastasis (CRLM). Unfortunately, only a minority of patients with CRLM are candidates for liver resection. The 5 -year survival of patients with liver-only disease and deemed unresectable is difficult to quantify but likely ranges around 5 to $10 \%$. Since 2013 , a few studies have shown that liver transplantation (LT) is feasible for selected patients with unresectable CRLM $(1,2)$. Recently, the Oslo University Hospital LT group has published their latest results with modified selection criteria and should be congratulated for their efforts. Nevertheless, the evidence available is still developing. For example, currently, there is a lack of robust evidence supporting the widespread use of LT for CRLM outside Norway, a particular region in terms of graft scarcity.

In 2013, Hagness et al. published the first series of patients with unresectable CRLM who underwent LT (1). The SECA-I trial was a prospective pilot study to assess the safety and effectiveness of LT for patients with unresectable CRLM. Details of the SECA-I design are presented in Table 1, along with its main results. Briefly, 25 patients were listed for LT, and 21 (84\%) underwent LT. After a median follow-up time of 27 months, none of the patients was disease-free. The disease-free survival (DFS) was $35 \%$ at 1 -year and $0 \%$ at 2-year. Interestingly, the overall survival (OS) was $95 \%$, $68 \%$, and $60 \%$ at 1-, 3- and 5-year. This difference between DFS and OS led the authors to evaluate recurrence patterns more closely. They identified that after LT, recurrences occurred at sites amenable for further treatment. For example, 7/21 (33\%) patients had lung-only recurrences and underwent ablation and/or surgical resection. In terms of safety, no patient died because of post-operative complications. Nonetheless, major complications (ClavienDindo IIIa) occurred in 10/21 (49\%) of their patients. Of note, four patients developed hepatic artery thrombosis and, of them, two required re-transplantation. The SECA-I trial demonstrated that LT for patients with unresectable CRLM is safe and effective. However, the rate of recurrences was high, which was the focus of criticism concerning the utility of LT for CRLM patients.

In their most recent study, entitled SECA-II, Dueland et al. assessed the impact of more restrictive selection criteria (3). The study presents the results of 15 patients with unresectable CRLM who underwent LT. The smaller population size reflects, likely, the more stringent inclusion criteria which included, in addition to the same inclusion criteria in the SECA-I trial: unresectable liver-only colorectal metastases, at least $10 \%$ response to systemic therapy, and at least 1-year between the primary tumor diagnosis and the listing for LT (Table 1). In comparison with the SECA-I trial, patients in SECA-II had a lower tumoral burden and carcinoembryonic antigen levels (Table 1). After a median follow-up of 36 months, 8/15 (53\%) patients developed disease recurrence. The DFS was $53 \%, 44 \%$, and $35 \%$ at 1-, 2- and 3-year, respectively. The OS was $100 \%, 83 \%$, and $83 \%$ at $1-, 3-$ and 5 -year, respectively. Authors reported the survival after recurrence. They have shown a $72 \%$ survival after recurrence at 4-years. In terms of complications, the rate of major complications 
Table 1 Details on design and main results from the SECA-I and SECA-II trials

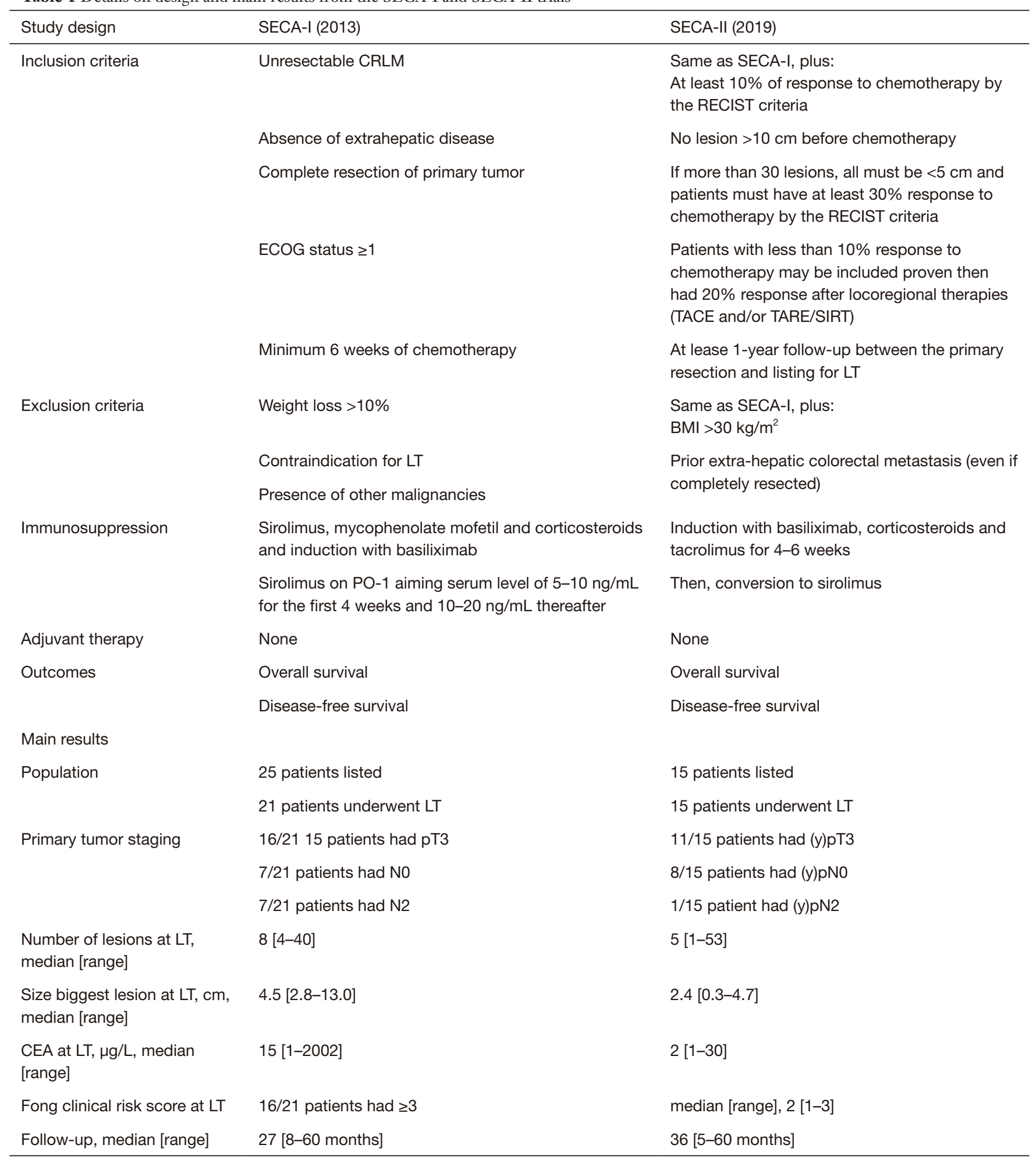

Table 1 (continued) 
Table 1 (continued)

\begin{tabular}{lll}
\hline Study design & SECA-I (2013) & SECA-II (2019) \\
\hline Overall survival & $95 \%$ 1-year & $100 \%$ 1-year \\
& $68 \%$ 3-year & $83 \%$ 3-year \\
& $60 \%$-year & $83 \% 5$-year \\
Disease-free survival & $35 \%$ 1-year & $53 \% 1$-year \\
& $0 \%$-year & $44 \%$ 2-year \\
Main conclusion & - & $35 \%$ 3-year \\
& LT is feasible for patients with unresectable CRLM & More restrictive selection criteria result in \\
\hline
\end{tabular}

BMI, body mass index; CEA, carcinoembryonic antigen; CRLM, colorectal liver metastasis; ECOG, Eastern Cooperative Oncology Group; LT, liver transplantation; PO, post-operative; SIRT, selective internal radiation therapy; TACE, transarterial chemotherapy; TARE, transarterial radioembolization.

was similar to the previous study: $47 \%$. In contrast, no patient required re-transplantation. Therefore, the SECA-II trial, successfully showed that more restrict selection criteria improve the outcomes. The main criticism, however, should be the short follow-up time. Of the 11 patients who were alive at the end of follow-up, only one had reached the 5 -year mark. This lack of longer follow-up, unfortunately, still prevents any strong conclusion from being drawn about the long-term benefit of LT for patients with CRLM.

The SECA-II trial sheds light on additional topics worthy of mention. The pre-LT examination with ${ }^{18} \mathrm{~F}$-FDG PET/CT has been shown as an option for patient selection. Assessing the ${ }^{18} \mathrm{~F}$-FDG PET/CT might identify tumors with less aggressive tumoral biology, as well as improve detection of extrahepatic disease (4). Negative nodal status on primary resection was associated with improved outcomes when compared to patients with $\mathrm{N}+$ disease. As seen in the SECA-I trial, patients with higher Fong Clinical Risk Score also had the worst outcomes in the SECA-II trial. The time between the primary colorectal surgery and LT deserves further investigation. In the SECA-II trial, the authors applied a mandatory 1-year follow-up period before LT. Finally, in SECA-II, patients were required to have a minimal response to chemotherapy to get listed for LT. Likely, these criteria will be applied in the future to select those patients who will benefit the most from LT.

The lack of a control group is the primary limitation in both SECA studies. The group from Oslo tried to compare the LT cohort with different cohorts of patients treated with palliative chemotherapy (5). The validity of such comparisons remains unclear. A prospective trial specifically designed to compare patients with similar baseline characteristics treated with LT and palliative chemotherapy alone would be ideal for demonstrating the precise benefit of LT. In this regard, two prospective trials are currently recruiting patients to compare outcomes after LT versus chemotherapy alone: in France, the TRANSMET trial (NCT02597348) and, in Norway, the SECA-III trial (NCT03494946). The inclusion of patients with unresectable CRLM in the LT waiting list would increase the scarcity of deceased donor grafts. In this regard, strategies to increase the donor pool are needed. This is even more important to consider when we note that in the SECA-I trial, two patients required re-transplantation. To increase the pool of grafts, the use of marginal grafts could be explored. The group from Oslo University Hospital has developed the RAPID concept: left lateral hepatectomy with left lateral segment graft implantation followed by completion hepatectomy after adequate graft volume has been achieved. The use of grafts from live donors would not impact on graft scarcity. Our group is currently recruiting patients with unresectable CRLM for live donor LT in a prospective trial (NCT02864485).

In conclusion, LT is feasible for patients with unresectable CRLM. The evidence from the SECAII trial shows that selection criteria are imperative; although, the best way to select these patients is still to be determined. The ideal criteria for patient selection would be a combination of tumoral biology (e.g., tumoral burden, serum CEA levels, ${ }^{18} \mathrm{~F}-\mathrm{FDG}$ PET/CT evaluation, etc.), response to previous treatments (either chemotherapy or locoregional) and time elapsed between the primary 
colorectal resection and the LT. Furthermore, each LT jurisdiction will have to adjust these criteria to its organ availability to minimize adversely impacting waitlist outcomes for other patients. Finally, the group from Oslo University Hospital has made a remarkable contribution to the transplantation community, but much remains to be clarified before LT for CRLM can be widely accepted.

\section{Acknowledgments}

Funding: None.

\section{Footnote}

Provenance and Peer Review: This article was commissioned by the editorial office, Hepatobiliary Surgery and Nutrition. The article did not undergo external peer review.

Conflicts of Interest: All authors have completed the ICMJE uniform disclosure form (available at http://dx.doi. org/10.21037/hbsn.2020.01.04). The authors have no conflicts of interest to declare.

Ethical Statement: The authors are accountable for all aspects of the work in ensuring that questions related to the accuracy or integrity of any part of the work are appropriately investigated and resolved.

Open Access Statement: This is an Open Access article distributed in accordance with the Creative Commons Attribution-NonCommercial-NoDerivs 4.0 International License (CC BY-NC-ND 4.0), which permits the noncommercial replication and distribution of the article with the strict proviso that no changes or edits are made and the original work is properly cited (including links to both the formal publication through the relevant DOI and the license). See: https://creativecommons.org/licenses/by-nc-nd/4.0/.

\section{References}

1. Hagness M, Foss A, Line PD, et al. Liver transplantation for nonresectable liver metastases from colorectal cancer. Ann Surg 2013;257:800-6.

2. Toso C, Pinto Marques H, Andres A, et al. Liver transplantation for colorectal liver metastasis: Survival without recurrence can be achieved. Liver Transpl 2017;23:1073-6.

3. Dueland S, Syversveen T, Solheim JM, et al. Survival Following Liver Transplantation for Patients With Nonresectable Liver-only Colorectal Metastases. Ann Surg 2020;271:212-8.

4. Grut H, Revheim ME, Line PD, et al. Importance of 18F-FDG PET/CT to select patients with nonresectable colorectal liver metastases for liver transplantation. Nucl Med Commun 2018;39:621-7.

5. Dueland S, Guren TK, Hagness M, et al. Chemotherapy or liver transplantation for nonresectable liver metastases from colorectal cancer? Ann Surg 2015;261:956-60.
Cite this article as: Gorgen A, Ivanics T, Sapisochin G. Liver transplantation for unresectable colorectal metastasis: a new hope. HepatoBiliary Surg Nutr 2020;9(5):665-668. doi: 10.21037/ hbsn.2020.01.04 